\title{
Damage Detection of 2D Frame Structures USING INCOMPLETE MEASUREMENTS BY Optimization Procedure AND Model Reduction
}

\author{
Du DINH-CONG ${ }^{1,3, *}$, Sang PHAM-DUY ${ }^{4}$, Trung NGUYEN-THOI ${ }^{2,3}$ \\ ${ }^{1}$ Division of Construction Computation, Institute for Computational Science, \\ Ton Duc Thang University, Ho Chi Minh City, Vietnam \\ ${ }^{2}$ Division of Computational Mathematics and Engineering, Institute for Computational Science, \\ Ton Duc Thang University, Ho Chi Minh City, Vietnam \\ ${ }^{3}$ Faculty of Civil Engineering, Ton Duc Thang University, Ho Chi Minh City, Vietnam \\ ${ }^{4}$ Faculty of Civil Engineering, Ho Chi Minh City University of Transport, Vietnam \\ *Corresponding Author: Du DINH-CONG (email: dinhcongdu@tdtu.edu.vn) \\ (Received: 11-September-2018; accepted: 19-October-2018; published: 31-October-2018) \\ DOI: http://dx.doi.org/10.25073/jaec.201823.203
}

\begin{abstract}
The article presents an effective method for damage assessment of $2 D$ frame structures using incomplete modal data by optimization procedure and model reduction technique. In this proposed method, the structural damage detection problem is defined as an optimization problem, in which a hybrid objective function and the damage severity of all elements are considered as the objective function and the continuous design variables, respectively. The teaching-learning-based optimization (TLBO) algorithm is applied as a powerful optimization tool to solve the problem. In addition, owing to the use of incomplete measurements, an improved reduction system (IRS) technique is adopted to reduce the mass and stiffness matrices of structural finite element model. The efficiency and robustness of the proposed method are validated with a 4-storey (3 bay) steel plane frame involving several damage scenarios without and with measurement noise. The obtained results clearly demonstrate that even the incompleteness and noisy environment of measured modal data, the present method can work properly in locating and estimating damage of the frame structure by utilizing only the first five incomplete modes' data.
\end{abstract}

\section{Keywords}

Damage Assessment, Frame Structure, Incomplete Modal Data, TeachingLearning-Based Optimization (TLBO), Model Reduction, Objective Function.

\section{INTRODUCTION}

During the long service period, the safety and functionality of structural systems in mechanical, civil and aerospace engineering may be severely affected by degradation and damage due to environmental conditions and unforeseen circumstances. In this context, structural health monitoring (SHM) has played a vital role in scrutinizing the healthy state of structural systems, and simultaneously making accurate maintenance decisions. Over the past decades, global damage detection techniques such as vibration-based damage identification (VBDI) techniques have attracted increasing attention, and a significant amount of research has been proposed and developed in the literature. For more details about these techniques, inter- 
ested readers may refer to excellent review articles [1]-[3].

From a mathematical viewpoint, VBDI problem can be regarded as an optimization problem, where the objective function is usually expressed by the discrepancies between the analytical data and measured data and the damage severity of elements is taken as design variables. For solving the optimization problem, meta-heuristic optimization algorithms as intelligent search methods have been widely adopted. Many researchers successfully applied meta-heuristic search algorithms such as genetic algorithm (GA) [4, particle swarm optimization (PSO) [5], differential evolution (DE) [6], imperialist competitive algorithm (ICA) [7 and ant colony optimization $(\mathrm{ACO})[8$ in the structural damage identification. More recently, Xu et al. [9] studied a method based on cuckoo search (CS) algorithm for structural damage detection. Nobahari [10] introduced an optimization method called echolocation search algorithm (ESA) for damage localization and estimation in trusses using changes in modal frequencies. Dinh-Cong et al. [11] presented a damage identification approach utilizing Jaya algorithm and hybrid objective function to find the damage sites and sizes of simple structures like truss and frames. Dinh-Cong et al. [12 proposed an efficient multi-stage optimization approach for damage assessment in plate-like structures using a modified differential evolution algorithm (MS-MDE).

From the above-mentioned researches, it is noted that although these algorithms were able to provide satisfactory results in locating and quantifying damaged elements, they were required to collect the complete modal data corresponding to an entire set of node/degree of freedom (DOF) of the structural finite-element (FE) model. This is inapplicable in real SHM systems owing to the cost of instrumentation and practical issues faced in the measurement data like that for rotational DOFs. Therefore, it is necessary and important to develop approaches for diagnosing the structural health state with measured incomplete modal data. For this purpose, there are a few strategies which have been dedicated to the research area. Several researchers used mode shape expansion techniques [13]-[15] and others employed model reduction techniques [16]-[18]. The objective of this work is to present a practical and workable method that attempts to deal with this challenge by applying a model reduction technique for reducing the initial $\mathrm{FE}$ model of a full structure to a reduced model relating to measured DOFs.

In this research, an effective method based on a combination of optimization procedure and model reduction technique is proposed for structural damage detection using incomplete measurements. In this proposed method, the damage identification problem is defined as an optimization problem, where a hybrid objective function and the damage extent of elements are considered as the objective function and the continuous design variables, respectively. For solving the optimization problem, the teachinglearning-based optimization (TLBO) algorithm, a novel population-based global search strategy, is utilized. This algorithm does not depend on any additional algorithm-specific control parameter [19. It has recently been introduced for dealing with various engineering applications and its results have outperformed when compared with those of other well-known optimization algorithms [19-[21]. In addition, due to the use of incomplete measurements, improved reduction system (IRS) technique [22] is adopted to condense structural physical properties (i.e. stiffness and mass matrices). The efficiency and robustness of the proposed method are validated with a 4-storey (3 bay) steel plane frame as a numerical example involving several damage scenarios without and with measurement noise. Furthermore, the accuracy of the proposed approach under the influence of noise in the incomplete measurements is also examined. The obtained results reveal that the proposed method can successfully determine the location and extent of damages in the frame structure with limited measuring points.

The reminders of this manuscript are then organized as follows. In section 2, we provide the background of IRS method. Section 3 introduces the cost function formulation and a brief description of TLBO algorithm. The obtained numerical results are discussed in section 4 . Finally, we draw the main conclusions in section 5 . 


\section{IMPROVED REDUCTION SYSTEM (IRS) TECHNIQUE}

For a linear behaving structure with $n$ DOFs, the undamped free vibration of the structure can be described as

$$
K \Phi_{j}=\omega_{j}^{2} M \Phi_{j}, \quad j=(1,2, \ldots, n)
$$

where $K$ and $M$ are the global mass and stiffness matrices of the structure $([n \times n])$, respectively; $\Phi_{j}$ and $\omega_{j}$ are the $j$-th mode shapes and natural frequency, respectively; and $n$ is the number of DOFs. Eq. (1) can be rewritten in the partitioned form as

$$
\begin{aligned}
& {\left[\begin{array}{cc}
\mathbf{K}_{m m} & \mathbf{K}_{m s} \\
\mathbf{K}_{s m} & \mathbf{K}_{s s}
\end{array}\right]\left\{\begin{array}{c}
\Phi_{m} \\
\Phi_{s}
\end{array}\right\}} \\
& =\omega_{j}^{2}\left[\begin{array}{cc}
\mathbf{M}_{m m} & \mathbf{M}_{m s} \\
\mathbf{M}_{s m} & \mathbf{M}_{s s}
\end{array}\right]\left\{\begin{array}{c}
\Phi_{m} \\
\Phi_{s}
\end{array}\right\}
\end{aligned}
$$

where the subscripts $s$ and $m$ represent the number of selected slave and master DOFs, respectively.

From the second row of the above equation, the following relationship between slave and master can be found as

$$
\Phi_{s}=\left(-\mathbf{K}_{s s}-\omega_{j}^{2} \mathbf{M}_{s s}\right)^{-1}\left(\mathbf{K}_{s m}-\omega_{j}^{2} \mathbf{M}_{s m}\right) \Phi_{m}
$$

In Guyan reduction technique [23, the inertia terms of Eq. (3) is neglected, leading to

$$
\left\{\begin{array}{c}
\Phi_{m} \\
\Phi_{s}
\end{array}\right\}=\left[\begin{array}{c}
\mathbf{I} \\
-\mathbf{K}_{s s}^{-1} \mathbf{K}_{s m}
\end{array}\right]=\mathbf{T}_{G} \Phi_{m}
$$

Substitution of Eq. (4) into Eq. (2), per multiplication by $\mathbf{T}_{G}^{T}$, one can be represented by a reduced eigenvalue problem as follows

$$
\left(\mathbf{K}_{R e}+\omega_{j, R e}^{2} \mathbf{M}_{R e}\right) \Phi_{j, R e}=\mathbf{0}
$$

where the reduced stiffness and mass matrices are, respectively, given by

$$
\mathbf{K}_{R e}=\mathbf{T}_{G}^{T} \mathbf{K} \mathbf{T}_{G}, \quad \mathbf{M}_{R e}=\mathbf{T}_{G}^{T} \mathbf{M} \mathbf{T}_{G}
$$

It is known that Guyan method is reliable only at zero frequency. To tackle this obstacle,
O'Callaghan 22 introduced a technique known as improved reduction system (IRS) method by considering the first order approximation of a binomial series expansion in the transformation of the slave DOFs. In the IRS method, the reduced stiffness $K_{\mathrm{IRS}}$ and mass $M_{\mathrm{IRS}}$ matrices can be obtained as

$$
\mathbf{K}_{I R S}=\mathbf{T}_{I R S}^{T} \mathbf{K} \mathbf{T}_{I R S}, \mathbf{M}_{I R S}=\mathbf{T}_{I R S}^{T} \mathbf{M} \mathbf{T}_{I R S}
$$

where

$$
\mathbf{T}_{I R S}=\mathbf{T}_{G}+\mathbf{S M} \mathbf{T}_{G} \mathbf{M}_{R e}^{-1} \mathbf{K}_{R e}
$$

with

$$
\mathbf{S}=\left[\begin{array}{cc}
\mathbf{0} & \mathbf{0} \\
\mathbf{0} & \mathbf{K}_{s s}^{-1}
\end{array}\right]
$$

\section{OPTIMIZATION- BASED STRUCTURAL DAMAGE DIAGNOSE PROBLEM}

Generally speaking, the structural fault diagnosis problem may be viewed as an optimization problem solved by an optimization technique. In this approach, damage identification process is commonly performed by minimizing an objective function in which the damage ratios of the elements can be considered as design variables. To obtain a set of damage variables containing the locations and sizes of damages, teaching-learning-based optimization (TLBO) algorithm is presented here as a powerful optimization tool. The section is dedicated to briefly present the foundation of this problem consisting of a hybrid objective function and TLBO algorithm.

\subsection{Hybrid objective function}

In this work, we use a hybrid objective function, proposed by Dinh-Cong et al. [12, is a combination of two different objective functions for 
finding the damage sites and severities [11. This objective function is given as

$$
\begin{aligned}
& f(\mathbf{x})=\frac{1}{2}(1-\operatorname{MDLAC}(\mathbf{x})+\mathrm{F}(\mathbf{x})), \\
& \mathbf{x}=\left(x_{1}, \ldots, x_{n}\right) \in[0,1]^{n}
\end{aligned}
$$

where

$$
\operatorname{MDLAC}(\mathbf{x})=\frac{|\Delta \mathbf{f} \delta \mathbf{f}(\mathbf{x})|^{2}}{\left(\Delta \mathbf{f}^{\mathrm{T}} \Delta \mathbf{f}\right)\left(\delta \mathbf{f}^{\mathrm{T}}(\mathbf{x}) \delta \mathbf{f}^{\mathrm{T}}(\mathbf{x})\right)}
$$

and

$$
\mathrm{F}(\mathbf{x})=\frac{1}{n m o d} \sum_{j}^{n c}\left(\frac{\left\|\mathbf{F}_{j}^{e x p}-\mathbf{F}_{j}^{a n a}(\mathbf{x})\right\|_{F r o}}{\left\|\mathbf{F}_{j}^{e x p}\right\|_{F r o}}\right)^{2}
$$

In Eq. 111, $x$ is a variable vector implying the size of damage of $n$ structural elements; $\delta \mathbf{f}(\mathbf{x})$ and $\Delta \mathbf{f}$ are, respectively, the analytical frequency change vector and the measured frequency change vector, which is expressed as

$$
\delta \mathbf{f}(\mathbf{x})=\frac{\mathbf{f}_{u d}-\mathbf{f}_{a n a}(\mathbf{x})}{\mathbf{f}_{u d}}, \quad \Delta \mathbf{f}=\frac{\mathbf{f}_{u d}-\mathbf{f}_{e x p}}{\mathbf{f}_{u d}}
$$

where $\mathbf{f}_{\text {ana }}(\mathbf{x})$ is the analytical natural frequencies containing the damage variable vector $x$; $\mathbf{f}_{\text {exp }}$ and $\mathbf{f}_{u d}$ are represented as the measured natural frequencies of the damaged and undamaged structures, respectively.

In Eq. $12, \mathbf{F}_{\exp }$ and $\mathbf{F}_{\text {ana }}(\mathbf{x})$ are, respectively, the flexibility matrix of damage model (experimentally measured) and analytical model corresponding to a set of damage variable vector $x$; nmod denotes the number of measured mode data (mode shapes and natural frequencies) ; $n c$ corresponds to the total number of columns in the flexibility matrix; and $\|\bullet\|_{F r o}$ denotes the Frobenius norm of flexibility change.

\subsection{Teaching-learning-based optimization algorithm}

Teaching-learning-based optimization (TLBO) algorithm is a novel and effective global search algorithm, first developed by Rao et al. in 2011 [19. This algorithm is motivated by the idea of traditional educational process in a classroom. The primary goal of TLBO algorithm is to apply these phases to increase the performance level of individuals and overall performance of the class. It has two vital components: a teacher and a group of learners (students), which is described through the process of exchanging information between the teacher and students. Firstly, students learn knowledge from their teacher, and then students also learn mutual interactions among themselves. In addition, the main advantage of the algorithm is that it does not have any intrinsic parameters. The searching process of TLBO algorithm is performed through the two operators, namely 'Teacher Phase' and 'Learner Phase', is briefly summarized in the following.

- Teacher phase: In the initial phase, the teacher who has the most knowledgeable person in the class tries to provide $\mathrm{him} /$ her knowledge to the learners in order to improve the average performance of all learners. Like any other populationbased meta-heuristic algorithm, the population in TLBO algorithm comprises a group of learners that is expressed as

$$
\begin{aligned}
& X_{i, G}=\left[\begin{array}{llll}
x_{i 1, G} & x_{i 2, G} & \ldots & x_{i n, G}
\end{array}\right] \\
& (i=1,2, \ldots, N P)
\end{aligned}
$$

where $x_{i n, G}$ is the $i$-th learner on $n$-th design variable in the $G$ iteration, and $N P$ denotes the number of learners (population size). After evaluating the fitness value of each learner, the student with the best fitness in that iteration is assigned as the teacher $\left(X_{i}^{\text {teacher }}\right.$ best,$\left.G\right)$ role, and the mean result of the class is defined by

$$
X_{j, G}^{\text {mean }}=\frac{1}{N P}\left[\begin{array}{llll}
\sum_{i=1}^{N P} x_{i 1} & \sum_{i=1}^{N P} x_{i 2} & \cdots & \sum_{i=1}^{N P} x_{i n}
\end{array}\right]
$$

Now, the teacher attempts to enhance the mean grade of the class by the transmission of their knowledge to all learners, as shown in the following expression

$$
\begin{gathered}
X_{j, i, G}^{\text {new }}=X_{j, i, G}^{\text {old }}+\operatorname{rand}\left(X_{i_{-} \text {best }, G}^{\text {teacher }}-T_{F} X_{j, G}^{\text {mean }}\right), \\
i=(1,2, \ldots, N P), \quad j=(1,2, \ldots, n)
\end{gathered}
$$


where $T_{F}=\operatorname{round}[1+\operatorname{rand}(0,1)\{2-1\}]$ is the teaching factor assigned a value of either 1 or 2 ; rand is a random number distributed within $[0,1]$. If the new locations (updated solution) $\left(X_{j, i, G}^{\text {new }}\right)$ show lower function value than the former one $\left(X_{j, i, G}^{\text {old }}\right), X_{j, i, G}^{\text {new }}$ are accepted and flowed to the next phase, otherwise $X_{j, i, G}^{\text {old }}$ is maintained.

- Learner phase: In this phase, every student improves its fitness value by randomly communicating with other different students. An individual learner gains new information from the other individuals who have more knowledge and experience than him/her. At any $G$ iteration, two students $X_{j, p, G}$ and $X_{j, k, G}$ are randomly selected, where $p \neq k$. The learning operator of students could be expressed as follows

$$
X_{j, p, G}^{\text {new }}=\left\{\begin{array}{l}
X_{j, p, G}+\operatorname{rand}\left(X_{j, p, G}-X_{j, k, G}\right) \\
\text { if } f\left(X_{j, p, G}\right)<f\left(X_{j, k, G}\right) \\
X_{j, p, G}+\operatorname{rand}\left(X_{j, k, G}-X_{j, p, G}\right) \\
\text { if } f\left(X_{j, k, G}\right)<f\left(X_{j, p, G}\right)
\end{array}\right.
$$

If the fitness value of $p$-th learner $\left(X_{j, p, G}^{n e w}\right)$ is better than that of the existing one $\left(X_{j, p, G}\right)$, it will be allowed to the next generation, otherwise $X_{j, p, G}$ will be unchanged in the population. The procedure of both the two operators is run until the stopping criterion is reached.

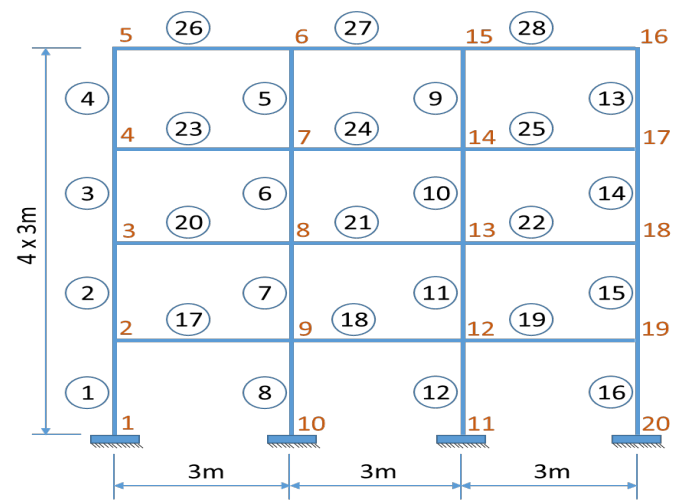

Fig. 1: Sketch of a 4-storey steel frame model.

\section{NUMERICAL EXAMPLE}

In this section, we consider a 4 -storey (3 bay) steel 2D frame [24], as shown in Fig. 1. The FE model of this structure is implemented by using two-dimensional frame elements that have two translational DOFs and one rotational DOF at each node. The whole FE model consists of 28 elements with 20 nodes. The cross-section of all members is equal to $A=0.04 \mathrm{~m}^{2}$. The mass density of material and modulus of elasticity are $\rho=7860 \mathrm{~kg} / \mathrm{m}^{3}$ and $E=200 \mathrm{GPa}$, respectively. Two damage scenarios including single and multiple damage sites are simulated. The details of both damage cases are summarized in Tab. 1. Here, damage is considered in form of a reduction in stiffness of selected members, i.e. $\mathbf{K}=\sum_{e=1}^{N e}\left(1-a^{e}\right) \mathbf{K}^{e}$, in which $\mathbf{K}^{e}$ and $\mathbf{K}$ are the stiffness matrix of the $e$-th element and the global stiffness matrix of damaged structure, respectively; and $a^{e}$ denotes the damage ratio of the $e$-th element that is bounded in the range $[0,1]$. In this study, the only first five natural frequencies and corresponding mode shapes are employed for the structural damage diagnosis problem.

In order to investigate the capability of the proposed approach, a comparison between the present TLBO algorithm and differential evolution (DE) algorithm is conducted for the two scenarios with and without measurement noise. Note that the common control parameters of two optimization algorithms involving the population size, maximum integration and stop criterion are set to 30,2000 and $10^{-6}$, respectively; whereas the remaining parameters of $\mathrm{DE}$ are referred to [25]. The added noise level on measured data is set to $\pm 5 \%$ noise in mode shapes and $\pm 1 \%$ noise in natural frequencies [12]. To fairly compare the robustness of the considered optimization algorithms, each algorithm is run 10 independent times for each test scenario.

It is generally considered that a good damage identification method is one which requires a minimum number of measured DOFs to conduct damage predictions in structures. In real practical situations, it is generally difficult and expen- 
Table 1. Single and triple damage scenarios induced in the steel 2D structure.

\begin{tabular}{cccc}
\hline Scenario 1 & \multicolumn{3}{c}{ Scenario 2 } \\
\hline Element No. & Damage ratio & Element No. & Damage ratio \\
\hline 12 & 0.25 & 01 & 0.20 \\
& & 8 & 0.30 \\
& & 21 & 0.20 \\
\hline
\end{tabular}

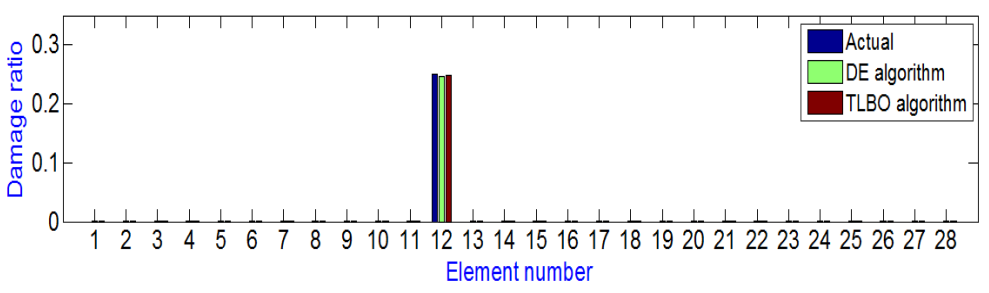

(a)

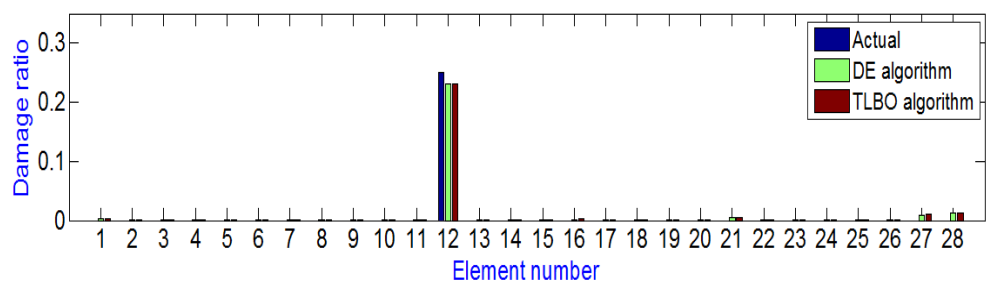

(b)

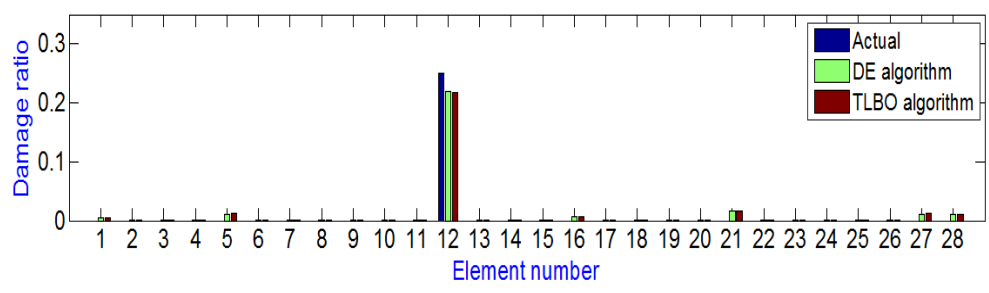

(c)

Fig. 2. Comparison of damage detection results obtained from two optimization algorithms for case 1 of the frame: (a) without noise, using 32 translational DOFs; (b) with noise, using 32 translational DOFs; (c) with noise, using 20 translational DOFs.

sive to measure rotational DOFs. To meet this requirement, in this present example, we assume that only translational DOFs could be measured. Thus, the translational DOFs are selected as the master DOFs that are considered for sensor installation to collect data. Here, for case study 1 , we supposed that all translational DOFs (32 DOFs) are measured, and then for case study 2 , only translational DOFs at nodes $2,3,4,5,6$,
$15,16,17,18$ and 19 are selected as 20 translational measured DOFs.

The average values of damage ratio of $28 \mathrm{el}-$ ements achieved by the two optimization algorithms for scenario 1 and 2 are reported in Fig. 2 and Fig. 3, respectively. The results show that in both case study, the assumed damages in the frame structure are localized properly by these two algorithms for both damage cases, element 


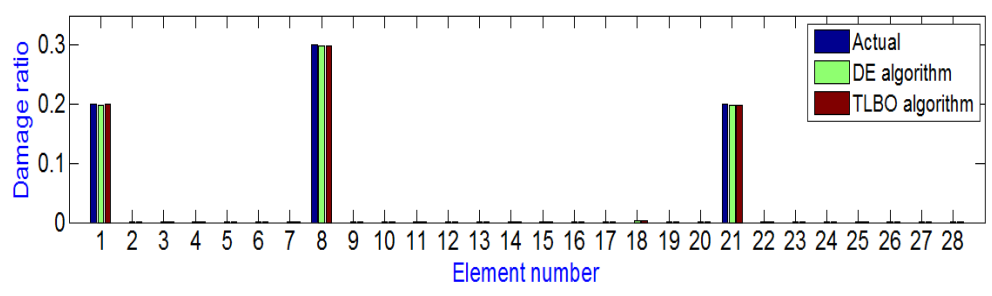

(a)

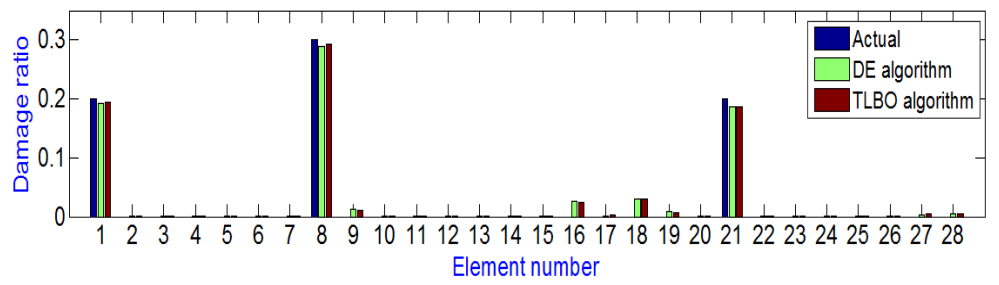

(b)

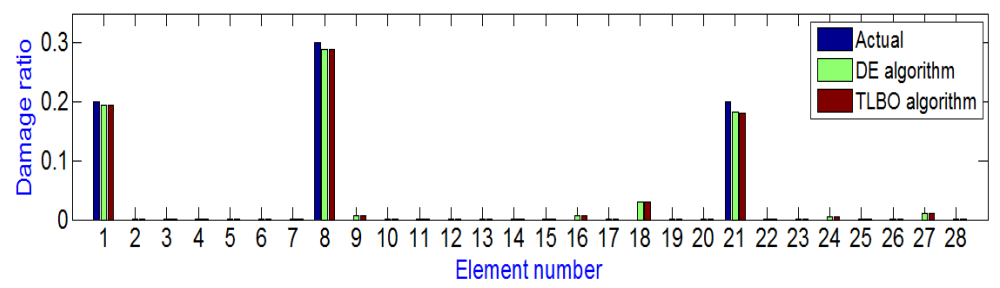

(c)

Fig. 3. Comparison of damage detection results obtained from two optimization algorithms for case 2 of the frame: (a) without noise, using 32 translational DOFs; (b) with noise, using 32 translational DOFs; (c) with noise, using 20 translational DOFs.

12 in case 1 and elements 1,8 and 21 in case 2. Also from these figures, one can find that: (1) the outcomes of both the two algorithms are very similar, (2) the presence of noise in measurement data lead to the decrease of the accuracy of damage identification results.

For further investigation of two chosen algorithm, the statistical results of damage estimation involving the mean value, standard deviation and the average number of structural analyses for both damage scenarios are summarized in Tab. 2. The statistical results indicate that even under case study 2 with noise-contaminated conditions, two algorithms can determine the degree of damaged elements with an acceptable accuracy. Particularly, for case study 1 with noise, the mean error between obtained average dam- age ratios and the actual damage ratios by the DE and TLBO algorithm are $5.94 \%$ and $5.63 \%$, respectively, while those for case study 2 with noise are $12.50 \%$, and $12.60 \%$, respectively. Besides, the standard deviation value of damage assessment results from the algorithms is relatively small. However, relating to computational cost, the required number of structural analyses of TLBO algorithm always works with much lower than that of $\mathrm{DE}$ algorithm. In addition, it is worth to mention that the errors in the obtained results can be increased when reducing the number of measured DOFs of the structure. 


\begin{tabular}{|c|c|c|c|c|c|c|c|c|c|}
\hline $\begin{array}{ll}\text { Table } & 2 . \\
\text { ure } & \text { for }\end{array}$ & $\begin{array}{l}\text { The } \\
\text { both }\end{array}$ & $\begin{array}{l}\text { statistical } \\
\text { damage }\end{array}$ & $\begin{array}{c}\text { results } \\
\text { scenarios }\end{array}$ & $\begin{array}{l}\text { of dam } \\
\text { with }\end{array}$ & $\begin{array}{l}\text { ge est } \\
\text { and }\end{array}$ & $\begin{array}{l}\text { mation } \\
\text { without }\end{array}$ & $\begin{array}{l}\text { in the } \\
\text { measur }\end{array}$ & $\begin{array}{l}\text { frame } \\
\text { ement }\end{array}$ & $\begin{array}{r}\text { struc- } \\
\text { noise }\end{array}$ \\
\hline & & & & $\begin{array}{c}\mathrm{DE} \\
\text { algorithm }\end{array}$ & & & $\begin{array}{c}\text { TLBO } \\
\text { algorithm }\end{array}$ & & \\
\hline Scenario & $\begin{array}{l}\text { Case } \\
\text { study }\end{array}$ & $\begin{array}{l}\text { Noise } \\
\text { level }\end{array}$ & $\begin{array}{l}\text { Actual } \\
\text { location }\end{array}$ & $\begin{array}{c}\text { Avg. } \\
\text { value }\end{array}$ & $\begin{array}{l}\text { Std. } \\
\text { Dev }\end{array}$ & $\begin{array}{l}\text { Avg. } \\
\text { NSA }\end{array}$ & $\begin{array}{l}\text { Avg. } \\
\text { value }\end{array}$ & $\begin{array}{l}\text { Std. } \\
\text { Dev }\end{array}$ & $\begin{array}{l}\text { Avg. } \\
\text { NSA }\end{array}$ \\
\hline \multirow{3}{*}{1} & 1 (32 DOFs) & $0 \%$ & $\alpha^{12}$ & 0.2471 & 0.0011 & 41259 & 0.2486 & 0.0005 & 6705 \\
\hline & & $5 \%$ & $\alpha^{12}$ & 0.2314 & 0.0321 & 45516 & 0.2320 & 0.0331 & 11455 \\
\hline & 2 (20 DOFs) & $5 \%$ & $\alpha^{12}$ & 0.2188 & 0.0373 & 48804 & 0.2179 & 0.0390 & 14555 \\
\hline \multirow{9}{*}{2} & & & $\alpha^{1}$ & 0.1987 & 0.0006 & & 0.1995 & 0.0003 & \\
\hline & & $0 \%$ & $\alpha^{8}$ & 0.2979 & 0.0011 & 40008 & 0.2989 & 0.0006 & 8230 \\
\hline & 1 (32 DOFs) & & $\alpha^{21}$ & 0.1989 & 0.0010 & & 0.1989 & 0.0008 & \\
\hline & & & $\alpha^{1}$ & 0.1930 & 0.0233 & & 0.1938 & 0.0237 & \\
\hline & & $5 \%$ & $\alpha^{8}$ & 0.2898 & 0.0211 & 49776 & 0.2929 & 0.0212 & 14830 \\
\hline & & & $\alpha^{21}$ & 0.1872 & 0.0176 & & 0.1867 & 0.0160 & \\
\hline & & & $\alpha^{1}$ & 0.1935 & 0.0167 & & 0.1936 & 0.0168 & \\
\hline & 2 (20 DOFs) & $5 \%$ & $\alpha^{8}$ & 0.2900 & 0.0230 & 47505 & 0.2895 & 0.0227 & 16635 \\
\hline & & & $\alpha^{21}$ & 0.1822 & 0.0189 & & 0.1812 & 0.0198 & \\
\hline
\end{tabular}

Avg. value $=$ average value of damage ratio with respect to $f$; Std. Dev $=$ standard deviation with respect to $f$; Avg. NSA = average number of structural analyses;

\section{CONCLUSIONS}

In this article, a damage diagnosis method based on a combination of optimization procedure and model reduction technique, which deals with incomplete modal data, is proposed. In the proposed approach, the damage detection process is conducted by minimizing a hybrid objective function solved via teaching-learning-based optimization (TLBO) algorithm. In addition, due to the use of incomplete modal data, improved reduction system (IRS) technique is adopted to condense the mass and stiffness matrices of the structure. To evaluate the performance of the proposed damage identification method, a 4storey (3 bay) steel plane frame as a numerical example involving single and multiple damage scenarios is conducted. Moreover, the presence of environmental noise in incomplete measurements on the accuracy of the proposed approach is also examined. Based on the presented results, some concluding remarks can be withdrawn as follows

- Both the TLBO and DE algorithms can offer quantitative estimation of damage locations and its levels with satisfactory precision, and also provide the same level of precision, regardless of incomplete and noisy- contaminated measurements. However, the TLBO algorithm always has the fewer number of structural analyses than the DE algorithm.

- The presented method is a simple and practical approach to the problem of damage localization and quantification in frame structures when limited measurement points are available. Nevertheless, the capability of the proposed method requires further studies for dealing with more complicated structures and an experimental test is necessary to find out how well the proposed method works under the effect of operational and environmental conditions.

\section{ACKNOWLEDGEMENT}

This research is funded by Vietnam National Foundation for Science and Technology Development (NAFOSTED) under grant number 107.02-2017.08.

\section{References}

[1] Carden, E. P., \& Fanning, P. (2004). Vibration based condition monitoring: a re- 
view. Structural health monitoring, 3(4), 355-377.

[2] Fan, W., \& Qiao, P. (2011). Vibrationbased damage identification methods: a review and comparative study. Structural health monitoring, 10(1), 83-111.

[3] Das, S., Saha, P., \& Patro, S. K. (2016). Vibration-based damage detection techniques used for health monitoring of structures: a review. Journal of Civil Structural Health Monitoring, 6(3), 477-507.

[4] Boonlong, K. (2014). Vibration-based damage detection in beams by cooperative coevolutionary genetic algorithm. Advances in Mechanical Engineering, 6, 624949.

[5] Nanda, B., Maity, D., \& Maiti, D. K. (2014). Modal parameter based inverse approach for structural joint damage assessment using unified particle swarm optimization. Applied Mathematics and Computation, 242, 407-422.

[6] Seyedpoor, S. M., Shahbandeh, S., \& Yazdanpanah, O. (2015). An efficient method for structural damage detection using a differential evolution algorithm-based optimisation approach. Civil Engineering and Environmental Systems, 32(3), 230-250.

[7] Masoumi, M., Jamshidi, E., \& Bamdad, M. (2015). Application of generalized flexibility matrix in damage identification using Imperialist Competitive Algorithm. KSCE Journal of Civil Engineering, 19(4), 994-1001.

[8] Majumdar, A., Nanda, B., Maiti, D. K., \& Maity, D. (2014). Structural damage detection based on modal parameters using continuous ant colony optimization. Advances in Civil Engineering, 2014(8), 1-14)

[9] Xu, H. J., Liu, J. K., \& Lu, Z. R. (2016). Structural damage identification based on cuckoo search algorithm. Advances in Structural Engineering, 19(5), 849-859.

[10] Nobahari, M., Ghasemi, M. R., \& Shabakhty, N. (2017). A novel heuristic search algorithm for optimization with application to structural damage identification. Smart Struct Syst, 19, 449-461.

[11] Du, D. C., Vinh, H. H., Trung, V. D., Hong Quyen, N. T., \& Trung, N. T. (2018). Efficiency of Jaya algorithm for solving the optimization-based structural damage identification problem based on a hybrid objective function. Engineering Optimization, 50(8), 1233-1251.

[12] Dinh-Cong, D., Vo-Duy, T., Ho-Huu, V., Dang-Trung, H., \& Nguyen-Thoi, T. (2017). An efficient multi-stage optimization approach for damage detection in plate structures. Advances in Engineering Software, 112, 76-87.

[13] Chen, S. H., \& Yang, X. W. (2000). Interval finite element method for beam structures. Finite elements in analysis and design, 34(1), 75-88.

[14] Yuen, K. V., Beck, J. L., \& Katafygiotis, L. S. (2006). Efficient model updating and health monitoring methodology using incomplete modal data without mode matching. Structural Control and Health Monitoring, 13(1), 91-107.

[15] Yuen, K. V. (2012). Updating large models for mechanical systems using incomplete modal measurement. Mechanical Systems and Signal Processing, 28, 297-308.

[16] Kourehli, S. S., Amiri, G. G., GhaforyAshtiany, M., \& Bagheri, A. (2013). Structural damage detection based on incomplete modal data using pattern search algorithm. Journal of vibration and control, 19(6), 821833.

[17] Hosseinzadeh, A. Z., Bagheri, A., Amiri, G. G., \& Koo, K. Y. (2014). A flexibility-based method via the iterated improved reduction system and the cuckoo optimization algorithm for damage quantification with limited sensors. Smart Materials and Structures, 23(4), 045019.

[18] Dinh-Cong, D., Vo-Duy, T., \& NguyenThoi, T. (2018). Damage assessment in truss structures with limited sensors using 
a two-stage method and model reduction. Applied Soft Computing, 66, 264-277.

[19] Rao, R. V., Savsani, V. J., \& Vakharia, D. P. (2011). Teaching-learning-based optimization: a novel method for constrained mechanical design optimization problems. Computer-Aided Design, 43(3), 303-315.

[20] Rao, R. V., \& Kalyankar, V. D. (2013). Parameter optimization of modern machining processes using teaching-learning-based optimization algorithm. Engineering Applications of Artificial Intelligence, 26(1), 524531 .

[21] Chauhan, S. J., \& Rodrigues, V. V. (2016). Teaching Learning Based Optimization (TLBO) for Optimal Placement of Piezo-Patches. Indian Journal of Science and Technology, 9(34), 1-5.

[22] O'CALLAHAN, J. (1989). A procedure for an improved reduced system (IRS) model. Proceedings of 7th IMAC, Las Vegas, NV., 17-21.

[23] Guyan, R. J. (1965). Reduction of stiffness and mass matrices. AIAA journal, 3(2), $380-380$.

[24] Mohan, S. C., Maiti, D. K., \& Maity, D. (2013). Structural damage assessment using FRF employing particle swarm optimization. Applied Mathematics and Computation, 219(20), 10387-10400.

[25] Vo-Duy, T., Ho-Huu, V., Dang-Trung, H., Dinh-Cong, D., \& Nguyen-Thoi, T. (2016). Damage detection in laminated composite plates using modal strain energy and improved differential evolution algorithm. Procedia engineering, 142, 182-189.

"This is an Open Access article distributed under the terms of the Creative Commons Attribution License, which permits unrestricted use, distribution, and reproduction in any medium, provided the original work is properly cited (CC BY 4.0)."

\section{About Authors}

D. DINH-CONG was born in Binh Dinh, Vietnam, 1987. He received the B.Sc. and MSc degree in Civil Engineering from Ho Chi Minh City Open University, Vietnam in 2011 and 2014, respectively. Currently, he works at Institute for Computational Science (INCOS), Ton Duc Thang University. His interests include structural health monitoring, optimization algorithms, model order reduction techniques and interval finite element methods.

S. PHAM-DUY received the B.Sc. and MSc degree in Civil Engineering from Ho Chi Minh City University of Technology, Vietnam in 2014 and 2017, respectively. Currently, he is a lecturer at Faculty of Civil Engineering - Ho Chi Minh City University of Transport, Vietnam. His interests include computational mechanics, reliability analysis of structures, structural health monitoring and optimization algorithms.

T. NGUYEN-THOI received the Ph.D degree in Mechanical Engineering, National University of Singapore (NUS), Singapore in 2010. He was appointed as a full professor of Ton Duc Thang University, Vietnam in 2017. Currently, he is director of Institute for Computational Science (INCOS), Ton Duc Thang University. His interests include computational mechanics, reliability analysis of structures, structural health monitoring, optimization algorithms, intelligent computation, structural optimization and applied optimization. 